\title{
A STUDY OF HAEMODYNAMIC RESPONSE TO LARYNGOSCOPY AND ENDOTRACHEAL INTUBATION WHEN USING FENTANYL:
}

KEY WORDS: Mobile phones, Health Care Workers, Microbial contamination

\section{Dr Brenda} Paulson*

Post-graduate, Department Of Anesthesiology, A.J.institute Of Medical Sciences, Mangalore. * Corresponding Author

Direct laryngoscopy is used to facilitate tracheal intubation under vision. Direct laryngoscopy and passage of a tracheal tube are noxious stimuli that can provoke adverse responses in the cardiovascular system. They cause intense reflex increase in heart rate, blood pressure and serum concentration of catecholamines. This study makes an effort to find the haemodynamic response to laryngoscope and endotracheal intubation using Fentanyl.

\section{INTRODUCTION:}

Direct laryngoscopy is used to facilitate tracheal intubation under vision. Direct laryngoscopy and passage of a tracheal tube are noxious stimuli that can provoke adverse responses in the cardiovascular system. They cause intense reflex increase in heart rate, blood pressure and serum concentration of catecholamines. The predominant response is tachycardia and arterial hypertension. The latter is due to increased cardiac output rather than increase in systemic vascular resistance and is associated with transient rise in central venous pressure.

Fentanyl is a synthetic opioid agonist used as an adjuvant to provide analgesia during general anaesthesia. Studies have shown its efficacy in reducing the hemodynamic response to laryngoscopy and endotracheal intubation. It acts by blunting the tracheal sensitivity to the stimulus of laryngoscopy and intubation..$^{2-6}$ This study makes an effort to find the haemodynamic response to laryngoscope and endotracheal intubation using Fentanyl.

\section{AIMS AND OBJECTIVES:}

To Study the haemodynamic response to laryngoscopy and endotracheal intubation when using Fentanyl.

\section{MATERIALS AND METHODS:}

This study was done from April 2018 to March 2019.

This study was done in the Department of Anesthesiology, A.J.Institute of Medical Sciences, Mangalore.

Patients were evaluated by taking detailed history, physical examination, airway assessment and relevant investigations preoperatively. They were asked to fast overnight. All patients received Inj.Fentanyl 1.5 microgram per $\mathrm{kg}$ intravenously 5 minutes prior to laryngoscopy.

A total of thirty patients were implemented for the study.

\section{RESULTS:}

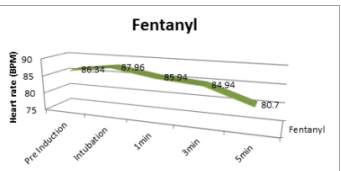

\section{Heart Rate:}

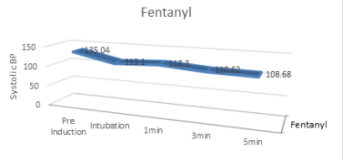

\section{Blood Pressure: Diastolic}

\section{DISCUSSION}

A hemodynamic response of increased HR and BP to manipulation in the area of the larynx, by means of laryngoscopy and intubation, has been well recognized for 60 years. Stimulation of mechanoreceptors in the pharyngeal wall, epiglottis, and vocal cords is thought to be the cause for the haemodynamic response. The receptors are abundant over arytenoid cartilage, vocal cords, epiglottis and hypopharynx. Transitory hypertension and tachycardia are probably of no consequence in healthy individuals, but either one or both may be hazardous to those with hypertension, myocardial insufficiency or cerebrovascular diseases. The transient changes can result in potentially deleterious effect like left ventricular failure, pulmonary edema, myocardial ischemia and cerebral haemorrhage.

Numerous studies have been published with different drugs to attenuate this response to laryngoscopy and intubation.

The average heart rate increased byl.85\% during laryngoscopy and intubation. According to a study ${ }^{7}$ they found Fentanyl effectively supressed the tachycardia during intubation. In 1970 the investigators used low dose of opioids as pre medications for blunting the laryngoscopic responses and observed significant reduction in the haemodynamic response to laryngoscopy and intubation. Use of morphine and fentanyl effectively reduced tachycardia and hypertension associated with laryngoscopy and intubation. However, they prolonged recovery time. The availability of synthetic narcotics like alfentanyl and sufentanyl effectively attenuated the pressor response to laryngoscopy and intubation. ${ }^{8}$

Another author studied the effects of fentanyl on cardiovascular response to laryngoscopy. The effect of fentanyl, $8 \mathrm{mcg} / \mathrm{kg}$. used as an adjunct to thiopental for induction of anesthesia, on the circulatory response to tracheal intubation was investigated in 36 patients undergoing major vascular surgery. Patients were randomly assigned to receive either thiopental, $6 \mathrm{mg} / \mathrm{kg}$, alone or thiopental, $3 \mathrm{mg} / \mathrm{kg}$, along with fentanyl, $8 \mathrm{mcg} / \mathrm{kg}$, for induction of anaesthesia. The electrocardiogram, arterial pressure, pulmonary capillary wedge pressure, cardiac output, and central venous pressure were measured during induction of anesthesia, laryngoscopy, and intubation. Mean arterial blood pressure increased more following intubation 
in patients given thiopental than in patients given fentanylthiopental, reaching a peak value of $144+/-4$ torr in patients receiving thiopental only, compared with $108+/-6$ torr in those receiving fentanyl and thiopental. Increases in systolic blood pressure, diastolic blood pressure, and pulmonary capillary wedge pressure with intubation were also significantly greater following administration of thiopental than following fentanyl-thiopental. There were no postoperative respiratory depression. They concluded that safely fentanyl blunts post intubation hypertension and tachycardia. $^{8}$

One more author ${ }^{9}$ also studied the effect of Fentanyl on the response to laryngoscopy and intubation. 100 surgical patients who required orotracheal intubation were randomly allocated into four groups. The first and third groups underwent fiberoptic intubation, in which anaesthesiologist inserted the endotracheal tube into the trachea under TV monitoring through a bronchoscope, and the second and fourth groups underwent conventional orotracheal intubation using a rigid laryngoscope. The third and fourth groups were pre treated with $2 \mathrm{mcg} / \mathrm{kg}$ fentanyl IV immediately before the induction of anaesthesia. Blood pressure and heart rate were measured noninvasively. A significant reduction in hemodynamic response was seen in only the group treated with fentanyl and intubated using the fiberoptic technique. Without fentanyl, there was no significant difference in hemodynamic changes between the groups. They concluded that, Fentanyl is useful in blunting the haemodynamic response to laryngoscopy and endotracheal intubation. More effective in blunting intubation response than that of laryngoscopy.

\section{CONCLUSION:}

1. Fentanyl is effective in controlling tachycardia in response to laryngoscopy and endotracheal intubation.

2. Fentanyl is effective in controlling the rise in blood pressue as a response to laryngoscopy and endotracheal intubation.

\section{REFERENCES:}

1. Batra Y, Mathew J. Airway management with endotracheal intubation. Indian J Anaesth. 2005;49(4):263-8.

2. King BD, Harris LC, Greifenstein FE, Elder JD, Dripps RD. Reflex circulatory responses to direct laryngoscopy and tracheal intubation performed during general anaesthesia.J New Eng Soc Anaesthesiol. 1951;12:556-66.

3. Ghaus MS, Kumar A, Wahal R, Bhatia VK, Agarwal J. A study of cardiovascular response during laryngoscopy and intubation and their attenuation by ultrashort acting beta blocker esmolol. Indian J Anaesth. 2002;46(2):104-6.

4. Howell SJ, Sear JW, Foëx P. Hypertension, hypertensive heart disease and perioperative cardiac risk. Br J Anaesth. 2004;92(4):570-83.

5. Lars RN, Roth J V, Hug CC, Nagle D. Esmolol Attenuates Hernodynamic Responses during Fentanyl-Pancuronium Anesthesia for Aortocoronary Bypass Surgery. Anesth Analg. 1986;65:451-6.

6. Liu PL, Gatt S, Gugino LD, Mallampati SR, Covino BG. Esmolol for control of increases in heart rate and blood pressure during tracheal intubation after thiopentone and succinylcholine. Can Anaesth Soc J. 1986;33(5):556-62.

7. Ranganathan S,Saravanan D, Harikumar S, Sumathi K. Attenuation of cardiovascular response during laryngoscopy and endotracheal intubation by using Fentanyl with Lignocaine.J Pharm biomed sci. 2013;27(14):508-14.

8. Feng CK, Chan KH, Liu KN, Or CH, Lee TY. A comparison of lidocaine, fentanyl, and esmolol For attenuation of cardiovascular response to laryngoscopy and tracheal intubation. Acta Anaesthesiol Sin. 1996 Jun;34(2):61-7.

9. Varma TARS, Aparanji K, Uma R. A Comparative Study of Efficacy of Esmolol and Fentanyl for Attenuation of Intubation Response during Laryngoscopy. J Evol MedDent Sci 2015;4(39) :6778-86 